\title{
The influence of tai chi and yoga on balance and falls in a residential care setting: a randomised controlled trial.
}

Padmapriya Saravanakumar

Candidate for $\mathrm{PhD}$

School of Nursing and Midwifery

Faculty of Health, University of Newcastle

Callaghan, Australia

Email: Padmapriya.Saravanakumar@uon.edu.au

Isabel Johanna Higgins

Professor, Older Person Nursing

School of Nursing and Midwifery

Faculty of Health, University of Newcastle, Callaghan,

Clinical Chair Hunter New England Local Health District, Australia

Email: Isabel.Higgins@newcastle.edu.au

Pamela Jane Van Der Riet

Associate Professor

School of Nursing and Midwifery

Faculty of Health, University of Newcastle

Callaghan, Australia

Email: Pamela.Vanderriet@newcastle.edu.au

Jodie Marquez

Lecturer, Discipline of Physiotherapy

Faculty of Health, University of Newcastle

Callaghan, Australia

Email: Jodie.Marquez@newcastle.edu.au

David Sibbritt (corresponding author)

Professor, Epidemiology

Faculty of Health, University of Technology Sydney

Broadway, New South Wales, Australia

Email: David.Sibbritt@uts.edu.au 


\section{Abstract}

Falls amongst older people is a global public health concern. Whilst falling is not a typical feature of ageing, older people are more likely to fall. Fall injuries amongst older people are a leading cause of death and disability. Many older people do not do regular exercise so that they lose muscle tone, strength, and flexibility which affect balance and predispose them to falls. The management of falls in residential care settings is a major concern with strategies for prevention and monitoring a focus in this setting. Yoga and tai chi have shown potential to improve balance and prevent falls in older adults. They also have potential to improve pain and quality of life.

The aim of this study was to determine the feasibility of conducting a 3-arm RCT with frail older people in a residential care setting to test the hypothesis that a 14 week modified tai chi or yoga program is more effective than usual care activity in improving balance function, quality of life, pain experience and in reducing number of falls.

There were no statistically significant differences between the three groups in the occurrence of falls. Yoga demonstrated a slight decrease in fall incidence; quality of life improved for the tai chi group. Only the yoga group experienced a reduction in average pain scores though not statistically significant.

The findings of the study suggest it is possible to safely implement modified yoga and tai chi in a residential care setting and evaluate this using RCT design. They show positive changes to balance, pain and quality of life and a high level of interest through attendance amongst the older participants. The results support offering tai chi and yoga to older people who are frail and dependent with physical and cognitive limitations. 
THE INFLUENCE OF TAI CHI AND YOGA ON BALANCE AND FALLS.

The influence of tai chi and yoga on balance and falls in a residential care setting: a randomised controlled trial.

Key words

Balance, Falls, Pain, Quality of life, Tai chi, Yoga 


\section{Introduction}

Falls amongst older adults is a global public health problem. Fall injuries continue to remain a leading cause of death and disability, especially for people aged 80 years and above (ABS, 2012; CDC, 2012). This poses a greater risk to residential aged care population with its largest proportion being people aged 85-89 years (AIHW, 2012; Caffrey et al., 2012; CPA, 2012). Fall injuries, if not fatal, could result in disability, fear of falling, delayed recovery, increased risk of subsequent falls, pain, sleep disturbances, depression, loss of independence and poor quality of life (Al-Faisal, 2006).

The increased vulnerability to falls and fall injuries in this population appears to be associated with age related alterations in body systems that lead to impaired balance function (Rubenstein, 2006; Vilijanen et al., 2009). Balance is defined as exertion of postural control that provides a stable base of support while remaining stationary or during movement (Winter et al., 1990). Balance impairment affects independence in activities of daily living, mobility and increases vulnerability to fall during the body's positional displacement (Lee \& Scudds, 2003). Balance and related measures such as coordination, proprioception, lower limb muscle strength and gait have been targeted by exercise interventions as means to prevent falls in older people.

Although exercise interventions are deemed vital to be effective components in fall prevention programs (Howe et al., 2012), careful consideration regarding the choice of exercise is warranted especially in frail older adults due to high fall risk (Cameron et al., 2010). Tai chi and yoga are two forms of complementary and alternative medicine (CAM) approaches that have recently emerged as being potentially beneficial in improving balance 
and thus preventing falls in older adults (Taylor-Piliae et al., 2010; Zettergren et al., 2011). Tai chi is a form of martial art and a low intensity exercise that is practiced with an emphasis on deep breathing, mental imagery, and slow, graceful movements to promote flexibility, balance and overall wellbeing (Wolf et al., 1996; Chyu et al., 2010). Yoga is a generic term for a physical, mental and spiritual discipline, the practice of which involves emphasis on postures (asanas), breathing techniques (pranayama) and meditation (dhyana) (Evans et al., 2009) and having positive effects on balance, muscle strength, endurance, flexibility and gait (Fran \& Chen, 2011; DiBenedetto et al., 2005).

Most studies that have reported benefits of tai chi and yoga have been conducted in relatively healthy community dwelling older adult population (Chen \& Tseng, 2008; Oken et al., 2006; Schmid et al., 2010; Li et al., 2004; Voukelatos et al., 2007). However inconclusive evidence exists on use of exercise interventions like tai chi and yoga for frail older people in residential care settings (Cameron et al., 2010). The high susceptibility to falls and fall injuries and the consequent magnitude of suffering highlight the need to investigate interventions that could potentially benefit the frail older population. In view of this, we aimed to investigate the feasibility of a randomised controlled trial that would compare the outcomes of participants randomised to three intervention groups, namely tai chi, yoga and usual care over a period of 14 weeks. We present the preliminary results from assessments in a small randomised cohort of older adults in a residential care facility $(\mathrm{RCF})$.

\section{Methods}

\section{Study design and setting}

This was a pilot randomised controlled trial (RCT) conducted in a residential aged care facility in New South Wales, Australia. The RCF was a 108 bedded facility offering low and 
high dependency residential care. This study was approved by the Human research ethics committee of University of Newcastle and by the ethics committee of the RCF. All participants provided written informed consent. The primary objective was to determine the feasibility of conducting a 3- arm RCT that would test the following hypothesis: a 14 week modified tai chi or yoga program is more effective than usual residential care facility activity program in improving balance function, quality of life, pain experience and in reducing number of falls. Feasibility studies are used to approximate the main components of a study (Arain, Campbell, Cooper \& Lancaster, 2010). In the case of this study, the feasibility criteria were: successful recruitment of 30 participants; $50 \%$ retention of participants until completion of study; $50 \%$ attendance of participants; and absence of serious adverse events requiring hospitalisation. The criteria for recruitment, retention and completion were based on the need to ensure an adequate number of participants for the intervention arms: yoga and tai chi, to test the outcome measures and to determine the suitability of yoga and tai chi with frail older people. The absence of serious adverse events was essential in the light of the need to demonstrate safety. The trial was registered with the Australian New Zealand Clinical Trials Registry and is reported following recommended guidelines for reporting results of pilot investigations by Thabane et al. (2010) and the CONSORT guidelines for randomised trials of non-pharmacological treatment (Boutron et al., 2008).

The trial was registered with the Australian New Zealand Clinical Trials Registry (ACTRN12612000103864). Data collection took place from February 2011 to June 2012. This pilot study is reported following recommended guidelines for reporting results of pilot investigations by Thabane et al. (2010) and the CONSORT guidelines for randomised trials of non-pharmacological treatment (Boutron et al., 2008). 


\section{Recruitment of study participants}

Study recruitment took place over 4 months from May to August 2011. Recruitment involved notices in the facility, the facility's newsletter, group presentations and individual meetings with staff and residents at the RCF.

\section{Randomization}

Permuted block randomisation with a block size of 6 was generated using MS office Excel. After baseline assessments, participants were randomly allocated to tai chi, yoga or usual care groups by a researcher not involved in recruitment who prepared the randomised list in sealed envelopes that were given to the facility staff a day before the commencement of the interventions. The randomization resulted in each group having 11 participants.

\section{Participants}

\section{Eligibility criteria}

The inclusion criteria were: people aged 60 and over, ability to; stand with support, understand English, understand and follow simple instructions and demonstrations, and the absence of severe debilitating illness, severe cognitive, hearing and visual impairment as determined by the RCF staff.

\section{Interventions}

Sessions were scheduled in the morning and were conducted by experienced and certified tai chi and yoga instructors. Both instructors were female with over 20 years of teaching experience in their modalities. The tai chi instructor, a retired physiotherapist, is in her 6os and volunteers as a tai chi instructor in several RAC facilities in her local area. The yoga instructor, in her 50's, has a Bachelor of Health Science in Complementary Medicine and a Diploma of Yoga Teaching. She also has her own yoga school and is a naturopath. 
The venue was a large, spacious, well lit, ventilated and air-conditioned room in the facility. Both tai chi and yoga programs were modified to suit the frail older participants, while progressing safely in complexity according to individual ability. Every participant had a sturdy chair behind them to rest when necessary and a solid four-footed frame; a rollator with breaks) in front to hold for support. For every session, there were at least four people to assist the participants and to provide safety and support when needed. The instructors explained and demonstrated the movements and postures, repeating instructions several times as necessary and at a pace suited for the participants. To facilitate better viewing, the participants were arranged in a semi-circle around the trainer. The instructors used techniques like providing clear, simple instructions, repeating demonstrations as necessary, mirroring movements (ie., instructor moving to her right when directing participants to move to the left), friendly approach, appreciation and maintained a good rapport throughout the entire intervention period.

\section{Tai chi}

The tai chi intervention consisted of a 14-week program of twice a week half-hour tai chi classes. Every session of the modified tai chi program began with warm-up exercises of different joints and progressed through 18 individual tai chi and qigong movement patterns, with repetitions for each pattern, using imagery, breathing and posture control. The sessions concluded with the lotus (AATC, 2010) that consisted of flowing relaxed movements that tell a story and a flow pattern of walking. The movements were slow, controlled and circular using functional patterns and engaging the mind. The modifications incorporated performance according to individual capability and safe level of comfort. Every participant had a sturdy chair behind them to rest when necessary and a solid four-foot frame (a rollator with breaks on) in front to hold on for support. Imagery from nature was used to describe and enhance the movement (e.g., a flying dove spreading wings). A calm and soothing oriental 
music was used throughout the class. Throughout the program a holistic approach was used emphasising good posture, natural breathing patterns, relaxation and balance.

\section{Yoga}

The yoga intervention consisted of a 14 week program of twice a week half-hour sessions. Every session commenced with awareness of breathing, slowing the breathing rate and limbering preparatory movements. This was followed by modified traditional yoga exercises, (asanas), breathing (pranayama), synchronising movements with breathing and yoga nidra, a type of relaxation. In order to be suitable for the frail participants, more sitting activities and limbering and preparatory movements were included. The yoga asanas; tadasana, urdhva hastasana, nitambasana, utkatasana, vrksasana, natarajasana, garudasana and virabhadrasana were performed while standing with two sturdy chairs, one behind the person (to rest whenever needed) and one in front to hold on at all times. The following asanas were performed seated: dandasana, marjariasana and matsyendrasana. The sessions concluded with pranayama and yoga nidra performed while the participants were seated comfortably, with the instructor guiding relaxation and mindful awareness of the body. A soothing music was played throughout the relaxation phase. The modified yoga program was intended to improve mobility, fluidity of movement, strength of muscles especially to stabilise the joints of the ankle and knee, integration of left and right hemispheres of the brain and body awareness.

\section{Usual care}

Whilst the control group was not offered any other program during the RCT the RCF encouraged all residents to access the Staying Active program with weekly half hour seated exercise sessions; Physical culture, games and group activities like bingo, group reading, story-telling, etc.; a gym with bikes, pulleys and massage by trained staff; assisted and independent activities such as walking, gardening, etc. The participants of the RCT were not 
prevented from accessing their usual care programs including the Staying Active program. Two participants (Tai Chi=1; Yoga=1) attended Physical culture during the intervention period.

\section{Outcome measures}

The primary outcome measure of the study was balance. The secondary outcome measures included fall incidence, pain and perceived quality of life.

\section{Balance}

Balance was measured using the Berg Balance Scale (Conradsson et al., 2007) by research assistants who were trained in the use of the tool and were blinded to participant allocation. Immediate pre and post intervention measurements were taken. The Berg balance scale is comprised of a series of 14 static and dynamic balance tasks each scored between $0-4$ giving a summed score between 0-56 with a higher score indicating better balance. There is consistent evidence of the reliability and validity of the Berg Balance Scale for older population in residential care (Donoghue et al., 2009; Conradsson et al., 2007).

\section{Fall incidence}

Falls were defined as "events that resulted in a person coming to rest inadvertently on the ground or floor or other lower level, excluding intentional change in position to rest in furniture, wall or other objects" (WHO, 2007). Fall incidence information was collected from the records maintained at the RCF. The data was collected for the period of six months preintervention, intervention period and 6 months post intervention period.

\section{Pain}

Pain was measured immediately pre and post intervention and mid intervention at week 7 using a verbal descriptor scale (VDS) validated for use with older people (Herr et al., 2004). The VDS comprised words that describe pain of increasing intensity such as: no pain, mild pain, moderate pain, severe pain, very severe pain, and most intense pain imaginable (Jones 
et al., 2005). Participants were asked to select the word that best described their pain. Scores of 0 to 6 were assigned to each description of pain (Ware et al., 2008).

\section{Quality of life}

Perceived quality of life was measured using the Dementia quality of life (DQOL) questionnaire, a tool that is found suitable and reliable for use in older adults with or without mild cognitive impairment (Moyle et al., 2012). This tool was administered immediately pre and post intervention and mid intervention at week 7 . The questionnaire comprised 29 items that were rated on five point Likert scales that measured frequency (ranging from never to very often) or enjoyment (ranging from not at all to a lot). Higher scores represented a better quality of life.

Data on pain and quality of life was collected by a researcher who was also a registered nurse. However, she was not blinded to participant allocation because of the funding limitations associated with this as a student project.

\section{Demographics and health status}

Demographic and health status data from residents' records were obtained as part of the initial screening process for recruitment. This included: age, medications, and medical history. The records were used to further note occurrence of any adverse events during the intervention period.

\section{Statistical analysis}

Analyses were performed using Stata 11 (StataCorp, 2009). Statistical analysis included chisquare test and Kruskal-Wallis one-way analysis of variance to ensure homogeneity to the three groups on baseline age, gender, number of medical diagnoses, number of prescribed drugs and outcome measures. A generalised estimating equation (GEE) model was used to compare the performance of the three groups over the 14 week period. All participant data were analysed per protocol method. 


\section{Results}

\section{Participant flow}

Out of 108 residents in an aged care facility, 49 expressed interests who were screened for participation in the study. Ten residents were found ineligible and six declined to participate before baseline measurements due to: 'inconvenience' $(n=3)$ or 'no reason given' $(n=3)$. Thirty three residents provided informed consent, completed baseline testing and were randomly assigned to each of the intervention groups (tai chi, yoga or usual care). The flow of participants through the study is represented in Figure 1.

\section{Baseline characteristics}

A greater proportion of participants were female $(72.7 \%)$ and the mean age was $83.8(\mathrm{SD}=$ 7.9) years with a range of 63-98. On average, the participants had 10 diagnosed medical conditions and had been prescribed between 4-22 medications. The median, range scores for the mini mental status examination scores (MMSE) were 27 (22-30). However, all participants could consent for themselves as verified by staff of the facility. Descriptive statistics are presented in Table 1. All groups were similar in the following characteristics: age, gender, cognitive status, number of medications and number of medical conditions. There were no significant differences between the groups at baseline for: balance, number of falls, pain or quality of life. None of the participants had current practice of tai chi or yoga although two participants from the yoga and tai chi groups recalled having practiced in the past tai chi and yoga respectively for a period of one to two years.

\section{Recruitment and follow up}

A total of 17 out of 22 people amongst the tai chi and yoga groups completed the intervention. The average attendance for the entire intervention period was greater for the tai chi group $(78.2 \%)$ than the yoga group $(76.3 \%)$. Five participants withdrew from the study 
during the intervention period ( 2 from the tai chi group and 3 from the yoga group) and three participants were lost to follow-up (1 from each group). The reasons for these losses were: the participants' concern about failing vision $(n=1)$, feeling unwell $(n=1)$, loss of interest $(n=1)$, no reason $(n=1)$, exclusion for safety reasons $(n=1)$ and death $(n=3)$. Control group participants had access to usual care; the Staying active program, physical culture and gym activities. As this was not monitored it is unknown how many control group members attended these.

\section{Adverse events}

One adverse event occurred during the study period. A participant of the yoga group experienced a fall during the session when he attempted to turn during a yoga posture. Although no injury was sustained, his participation in the study was ceased as it was revealed that he suffered from Meniere's disease (CHEG, 1995).

\section{Effects on primary outcome: balance}

The yoga group was the only group to demonstrate an improvement in average Berg balance scores which improved from 34.0 to 42.8 post-intervention. In contrast, both the tai chi and control group showed a decline in balance measures immediately post-intervention, with a difference in average of -3.0 and -3.4 respectively (Table 2). However, the difference in balance score between the groups was not statistically significant.

\section{Effects on secondary outcomes: fall incidence, quality of life and pain}

There were no statistically significant differences between the three groups in the occurrence of falls. However, only the yoga group demonstrated a slight decrease in fall incidence from 11 in the pre-intervention period to 9 in the post intervention period. While pre- and postintervention mean fall incidents remained similar for the tai chi group, they rose from 0.8 to 2.6 for the control group. All three groups had lower mean fall incidents during the 14 week intervention period, with the tai chi group having no fall incidents (Table 2). 
Though not statistically significant, perceived quality of life improved solely for the tai chi group from an average of 89.7 at baseline to 96.6 at week 14, whereas it remained almost the same for the yoga group (93.9 at baseline and 94.0 at week 14) and reduced by 2.4 for the control group. Of the three groups, only the yoga group experienced a (non-statistically significant) reduction in average pain scores (from 2.5 to 2.1 ). Both the tai chi and control groups had an increased pain score average post intervention (Table 2).

The GEE models indicated no statistically significant differences in any of the outcome measures between the three groups. However, note that post-hoc power calculations revealed that our study had $\leq 32 \%$ statistical power to detect differences between the control group and intervention groups for all four outcomes measures.

\section{Discussion}

In this pilot study, we demonstrated the feasibility of conducting a RCT that compared modified programs of yoga and tai chi in residential care settings. All the feasibility criteria set out were met: 33 participants were recruited, More than 50\% of participants of the yoga and tai chi intervention groups had an attendance of $\geq 70 \%$ and 28 participants completed the trial. There were no serious adverse events associated with participation in the tai chi and yoga programs.

Our study represents the first ever examination into whether a modified tai chi or yoga program could be safely implemented in a residential care setting. Although not conclusive, our findings suggest that tai chi and yoga may be associated with improvement in balance, pain and quality of life in frail older people. Improvement in balance has been reported in previous studies that used yoga for relatively robust older adults living in the community (Zettergren et al., 2011; Chen \& Tseng, 2008; Oken et al., 2006; Schmid et al., 2010). The 
favourable effect of yoga intervention on balance is hypothesized to be mediated by the slow stretching postures that increase muscle strength and endurance (Fan \& Chen, 2011), improving flexibility (Schmid et al., 2010) and gait function (DiBenedetto et al., 2005). Two quasi experimental studies that involved transitionally frail older adults in long term care facilities in Taiwan (Fan \& Chen, 2011; Chen et al., 2010) found similar results with silver yoga exercises. Although the participants in these two studies were described as transitionally frail, they were able to assume challenging positions such as kneeling, squatting, supine and prone on the floor. In contrast, the participants of our study had a mean BBS of 32.7( $\mathrm{SD}=13.9)$, which was substantially lower than the published normative values for similarly aged community dwelling adults (males: 53, females 50) (Steffen et al., 2002).

Randomised controlled trials that employed tai chi as the intervention have reported significant improvement in balance function, reduced fear of falling and fall risk in older adults who were in reasonably good health and community dwelling (Taylor-Piliae et al., 2010; 2004; Voukelatos et al., 2007). Similar results were reported by two studies where the older participants had high risk of falls (Taylor et al., 2012; Tousignant et al., 2012). In contrast, the tai chi and control groups in our study experienced a decline in balance. This outcome could be due to the frail and dependant nature of the participants or the lack of challenge to balance as the tai chi participants spent less time collectively, performing standing exercises than the yoga participants. This association has been reported in recent systematic reviews (Howe et al., 2012; Cameron et al., 2010). The low Berg balance scores of our participants place them in a high falls risk classification whereby a score of $\leq 40$ has been associated with an almost 100\% fall risk (Shumway-Cook et al., 2010). Our results for balance and falls are congruous in that only the yoga group experienced a decrease in fall incidents and concomitant increase in berg balance scores. This finding supports previously reported association between berg balance scores and falls (Chiu et al., 2003). It could be 
argued that the change scores demonstrated in the yoga participants may constitute clinically valuable change. A recent systematic review (Downes et al., 2013) suggests that if an individual experiences a change of between 3 and 7 points one can be $95 \%$ confident that there has been a real change in balance. Given the improvement in average score of 3.2 points following 14 weeks of yoga, this positive finding warrants further investigation.

Our analysis revealed that the pain scores measured by VDS improved in the yoga group although not statistically significant. However, the improvement of 0.6 points measured by VDS is considered to be a clinically significant difference (Edelen \& Salibay, 2010; Kendrick \& Strout, 2005). This is an important finding considering the high prevalence (80\%) and frequency of pain that could further exacerbate frailty in institutionalised older adults who suffer multiple ailments and co-morbidities (Jacobsson et al., 2003; Malec et al., 2012). A recent systematic review reported effectiveness of yoga in significantly reducing pain over control interventions like therapeutic exercises, touch and manipulation, relaxing yoga, selfcare, standard care or no intervention in nine out of 10 RCTs (Posadzki et al., 2011). The mindfulness approach of yoga is hypothesised to affect hyper vigilance and emotional reactivity aspects of chronic pain by shifting the person's attention away from painful stimuli while increasing awareness of the body (Hassed, 2013).

The tai chi group had improved DQOL scores post intervention, this finding being consistent with previous studies (Chyu et al., 2010; Lee et al., 2009). The yoga group had similar scores pre- and post-intervention, while the control group not only had no improvement, but had scores lower than pre-intervention. Perceived quality of life scores could be influenced by factors other than the interventions themselves, such as: social inclusion, enjoying participation in group activity, distraction, novelty of the activity, a purposeful feeling (something to look forward to), promotion of positive attitude, self-efficacy, etc. 
We found that consistent support of the management and staff of the facility was instrumental in conducting the study. The frail nature of residents, their high level of dependency and the shortage of nursing staff added to the challenge of introducing a novel intervention for the purpose of testing its suitability in a trial such as ours. Presence of additional volunteers and staff enabled safe conductance of the programs. Frequent visits and volunteering at the facility helped the researcher to develop a good rapport with the residents. Researchers who measured outcomes had to reschedule visits several times due to unavailability of participants owing to illness, visits to doctors and other appointments.

Both the tai chi and yoga groups had more than $75 \%$ average attendance, in spite of the frail and dependent nature of the participants. Enthusiasm in participation was observed in most participants. Some participants $(n=5)$ who initially discontinued participation due to health and personal reasons (hospitalisation, death of a family member) willingly continued to attend the tai chi and yoga sessions after returning to the residential facility.

There are several limitations to this study that necessitate discussion. The primary aim of this study was to assess the feasibility of conducting a randomised controlled trial comparing tai chi and yoga as fall prevention interventions in residential care settings. The small sample size and underpowered nature of the study renders the findings as preliminary and incapable of making definitive conclusions on effectiveness and safety of the interventions tai chi and yoga. Further, $36.4 \%$ of participants had MMSE score of $\leq 25$, indicating possible mild cognitive impairment (Vertesi et al., 2001). Therefore, we cannot comment on the ability of residents with moderate and severe cognitive impairment safely participating in these interventions. Measures of physical activity of the three groups were not precise. The RCF had provisions such as gym, massage, physical culture, a weekly exercise activity and gardening activities. Participants of all groups had access to all these activities during the intervention period. Hence, potential confounding by differences in physical activity between 
intervention and control groups could not be adequately controlled. Blinding was impossible for the participants who were from the same facility and for the trainers who delivered the interventions. The researcher who measured outcomes pain and quality of life was also not blinded to treatment allocation of the participants. This could have been a potential source of bias.

There are several implications for nursing and practice arising from this study. The findings show it is possible to safely implement yoga and tai chi in residential care with older people who are frail, and dependent with physical and cognitive limitations. They also show that yoga and tai chi may be important in the prevention of falls in older people. Whilst that yoga and tai chi are viable alternatives to the usual suite of activities offered to people in residential care settings, as with any new initiative, it is essential to have the support of management staff, nurses and carers. For these activities they needed dedicated time, space and support. Frail older people need assistance to mobilise and attend the sessions. For safety there needed to be a large room with; warmth, quiet, privacy, space for movement, appropriate chairs and distance between chairs, and physical supports such as walking frames and a small number of dedicated staff. The care staff needed to be open to the idea of yoga and tai chi, flexible, empathetic and willing to facilitate attendance of older people to sessions on time. The high level of interest for yoga and tai chi amongst the older participants needs to be explored. What were the perceptions and experiences of the older participants? What other supported activities might be possible? For example, might table tennis, ping pong, indoor golf and bowls be viable alternatives to usual care? How might these compare with interactive games offered in the form a Wiis? The management and nursing staff of RACFs need to be open to exploring possibilities for new and innovative alternatives with older people, in particular those that might help to prevent falls, enhance social contact, and promote health and wellbeing. In addition, they need to explore ways of breaking down 
barriers such as care routines, and perceptions about physical ability, frailty and the limitations of cognition.

\section{Conclusion}

In conclusion, we remain optimistic that modified tai chi and yoga programs may constitute an important CAM approach to fall prevention in residential care settings. With this in mind, this study needs to be replicated in a large randomised multi centred controlled clinical trial to assess the external validity of these results. An economic evaluation also needs to be undertaken as it is evident from the present study that implementation of yoga and tai chi depends on a range of resources, however the costs of these may outweigh the costs from the sequelae of falls in older people. The staff members' attitude and knowledge about the yoga and tai chi programmes as well as their resources, interest, and commitment to mobilising the residents were important adjuncts to this trial. RACF staff need to be aware of the potential for yoga and tai chi as alternative forms of physical activity for older people, and the impact these may have on improving their quality of life, overall wellbeing, pain and in preventing falls.

\section{Acknowledgement}

The authors are grateful to all the residents, staff and management of the residential aged care facility of Uniting Care, New South Wales, Australia for their support to this research project.This research was supported through an AusAID Leadership Award provided to the lead author as part of her Doctoral studies. 


\section{References}

Al-Faisal, W. (2006) Falls Prevention for Older Persons : Eastern Mediterranean Regional Review, World Health Organisation.

Australian academy of tai chi (AATC) (2010) Retrieved from [http://www.livingchi.com.au/wpcontent/uploads/2010/07/Lotus-Qigong.pdf]

Australian Bureau of Statistics (ABS) (2012) Causes of death, Australia 2010 (No.3303.0): Australian Bureau of Statistics.

Australian Institute of Health and Welfare (AlHW) (2012) Residential aged care in Australia 2010-11: a statistical overview (No. AGE 68). In: 2012; Canberra: AlHW.

Boutron, I., Moher, D., Altman, D. G., Schulz, K. F., Ravaud, P., \& CONSORT Group (2008). Extending the CONSORT statement to randomized trials of nonpharmacologic treatment: explanation and elaboration. Annals of Internal Medicine, 148(4), 295-309.

Caffrey, C., Sengupta, M., \& Park-Lee, E. (2012)Residents living in residential care facilities:United States, 2010. NCHS data brief, no 91. In. Hyattsville, MD: National Center for Health Statistics.

Cameron, I. D., Murray, G. R., Gillespie, L. D., Robertson, M. C., Hill, K. D., Cumming, R. G., \& Kerse, N. (2010) Interventions for preventing falls in older people in nursing care facilities and hospitals. Cochrane Database of Systematic Reviews. Chichester, UK: John Wiley \& Sons, Ltd.

Centre for Policy on Ageing (CPA) (2012) A profile of residents in Bupa care homes: results from the 2012 Bupa census. London: Centre for Policy on Ageing.

Centers for Disease Control and Prevention (CDC) (2012) Leading Causes of Death Reports, National and Regional, 1999-2010. Injury Prevention and Control: data and statistics (WISQARS).

Chen, K-M. M., Fan, J-T. T., Wang, H-H. H., Wu, S-J. J., Li, C-H. H., \& Lin, H-S. S. (2010) Silver yoga exercises improved physical fitness of transitional frail elders. Nursing Research, 59; 364-370.

Chen, K-M. M., \& Tseng, W-S. S. (2008) Pilot-testing the effects of a newly-developed silver yoga exercise program for female seniors. The journal of nursing research, 16(1), 37-46.

Chiu, A. Y. Y., Au-Yeungg, S. S. Y., \& Lo, S. K. (2003) A comparison of four funtional tests in discriminating fallers from non-fallers in older people. Disability \& Rehabilitation, 25(1), 45-50.

Chyu, M. C., James, C. R., Sawyer, S.F., Brismee, J. M., Xu, K. T., Poklikuha, G., Dunn, D.M., \& Shen, C. L. (2010) Effects of tai chi exercise on posturography, gait, physical function and quality of life in postmenopausal women with osteopaenia: a randomized clinical study. Clinical Rehabilitation, 24(12), 1080-1090.

Committee on Hearing and Equilibrium guidelines for the diagnosis and evaluation of therapy in Meniere's disease (CHEG). (1995) Otolaryngology - Head and Neck Surgery, 113(3):181-185.

Conradsson, M., Lundin-Olsson, L., Lindelöf, N., Littbrand, H., Malmqvist, L., Gustafson, Y., \& Rosendahl, E. (2007) Berg Balance Scale: Intrarater Test-Retest Reliability Among Older People Dependent in Activities of Daily Living and Living in Residential Care Facilities. Physical Therapy, 87(9), 1155-1163. 
DiBenedetto, M., Innes, K. E., Taylor, A. G., Rodeheaver, P. F., Boxer, J.A., Wright, H. J., \& Kerrigan, D. C. (2005) Effect of a gentle lyengar yoga program on gait in the elderly: an exploratory study. In: Archives of Physical Medicine and Rehabilitation, 86, 1830-1837.

Donoghue, D., Phyiotherapy Research and Older People (PROP) group, \& Stokes, E,K. (2009) How much change is true change? The minimum detectable change of the berg balance scale in elderly people. Journal of Rehabilitation Medicine, 343-346.

Downes, J., Marquez, J., \& Chiarelli, P. (2013) The Berg balance scale has high intra and inter rater reliability but absolute reliability varies across the scale: a systematic review. Journal of physiotherapy, 59(2), 93-99.

Edelen, M. O., \& Saliba, D. (2010) Correspondence of verbal descriptor and numeric rating scales for pain intensity: an item response theory calibration. Journals of Gerontology Series A-Biological Sciences \& Medical Sciences, 65(7), 778-785.

Evans, S., Tsao, J. C. I., Ternlieb, B. S., \& Zeltzer, L. K. (2009) Using the biopsychosocial model to understand the health benefits of Yoga. Journal of Complementary and Integrative Medicine, 6, 1-21.

Fan, J-T., \& Chen, K-M. (2011) Using silver yoga exercises to promote physical and mental health of elders with dementia in long-term care facilities. International Psychogeriatrics, 23, 12221230.

Hassed, C. (2013) Mind-body therapies: Use in chronic pain management. Australian Family Physician, 42(1/2).

Herr, K. A., Spratt, K., Mobily, P. R., \& Richardson, G. (2004) Pain intensity assessment in older adults: use of experimental pain to compare psychometric properties and usability of selected pain scales with younger adults. Clinical Journal of Pain, 20(4), 207-219.

Howe, T. E., Rochester, L., Neil, F., Skelton, D. A., Ballinger, C., Skelton, D. A. (2012) Exercise for improving balance in older people. Cochrane Database of Systematic Reviews. Chichester, UK: John Wiley \& Sons, Ltd.

Jakobsson, U., Klevsgård, R., Westergren, A., \& Hallberg, I. R. (2003) Old people in pain: A comparative study. Journal of Pain and Symptom Management, 26(1), 625-636.

Jones, K. R., Fink, R., Hutt, E., Vojir, C., Pepper, G. A., Scott-Cawiezell, J., \& Mellis, B. K. (2005) Measuring pain intensity in nursing home residents. Journal of Pain \& Symptom Management, 30(6), 519-527.

Kendrick, D. B., \& Strout, T. D. (2005) The minimum clinically significant difference in patientassessed numeric scores for pain. American Journal of Emergency Medicine, 23, 828-832.

Lee, H. K. M., \& Scudds, R. J. (2003) Comparison of balance in older people with and without visual impairment. Age \& Ageing, 32(6), 643-649.

Lee, L. Y. K., Lee, D. T.F., \& Woo, J. (2009) Tai chi and health related quality of life in nursing home residents. Journal of Nursing Scholarship, 41(1), 35-43. 
Li, F., Harmer, P., Fisher, K. J., \& McAuley, E. (2004) Tai Chi: improving functional balance and predicting subsequent falls in older persons. Medicine and Science in Sports and Exercise, 36(12), 2046-2052.

Malec, M., Knoebel, R. W., \& Shega, J. W. (2012) Noncancer pain management in seriously ill older adults: practical considerations with opioid use. Clinical Geriatrics.

Moyle, W., Gracia, N., \& Murfield, J. E. (2012) Assessing quality of life of older people with dementia in long-term care: a comparison of two self-report measures. Journal of Clinical Nursing, 21(11-12), 1632-1640.

Oken, B. S., Zajdel, D., Kishiyama, S., Flegal, K., Haas, M., Kraemer, D. F., Lawrence, J., \& Leyva, J. (2006) Randomized, controlled, six-month trial of yoga in healthy seniors: effects on cognition and quality of life. Alternative Therapies in Health and Medicine, 12(1), 40-47.

Posadzki, P., Ernst, E., Terry, R., \& Lee, M. S. (2011) Is yoga effective for pain? A systematic review of randomized clinical trials. Complementary Therapies in Medicine, 19(5), 281-287.

Rubenstein, L. Z. (2006) Falls in older people: epidemiology, risk factors and strategies for prevention. Age \& Ageing, 35, ii37-ii41.

Schmid, A. A., van Puymbroeck, M., \& Koceja, D. M. (2010) Effect of a 12-week yoga intervention on fear of falling and balance in older adults: a pilot study. Archives of Physical Medicine and Rehabilitation, 91(4), 576-583.

Shumway-Cook, A., Baldwin, M., Polissar, N. L., \& Gruber, W. (1997) Predicting the probability for falls in community-dwelling older adults, Physical Therapies, 77(8), 812-819.

StataCorp. (2009) Stata Statistical Software: Release 11. College Station, TX: StataCorp LP.

Steffen, T. M., Hacker, T. A., \& Mollinger, L. (2002) Age- and gender-related test performance in community-dwelling elderly people: Six-minute walk test, Berg Balance Scale, Timed Up \& Go Test,a nd gait speeds. Physical Therapy, 82(2), 128-137.

Taylor, D., Hale, L., Schluter, P., Waters, D. L., Binns, E. E., McCracken, H., McPherson, K., \& Wolf, S.L. (2012) Effectiveness of Tai Chi as a Community-Based Falls Prevention Intervention: A Randomized Controlled Trial. In: Journal of American Geriatrics Society, 60, 841-848.

Taylor-Piliae, R. E., Newell, K. A., Cherin, R., Lee, M. J., King, A. C., \& Haskell, W. L. (2010) Effects of Tai Chi and Western exercise on physical and cognitive functioning in healthy communitydwelling older adults. Journal of Aging \& Physical Activity, 18(3), 261-279.

Thabane, L., Ma, J., Chu, R., Cheng, J., Ismaila, A., Rios, L., Robson, R., Thabane, M., \& Goldsmith, C. A (2010) tutorial on pilot studies: The what, why and how. BMC Medical Research Methodology, $10,1$.

Tousignant, M., Corriveau, H., Roy, P-M., Desrosiers, J., Dubuc, N., Hébert, R., Tremblay-Boudreault, V., \& Beaudoin, A-J. (2012) The effect of supervised Tai Chi intervention compared to a physiotherapy program on fall-related clinical outcomes: A randomized clinical trial. Disability and Rehabilitation, 34, 196-201. 
Vertesi, A., Lever, J. A., Mollo, D. W., Sanderson, B., Tuttle, I., Pokoradi, L., \& Principi, E. (2001) Standardized mini-mental state examination. use and interpretation. Can Fam Physician, 47, 2018-2023.

Viljanen, A., Kaprio, J., Pyykko, I., Sorri, M., Pajala, S., Kauppinen, M., Koskenvuo, M., \& Rantanen, T. (2009) Hearing as a predictor of falls and postural balance in older female twins. In: The journals of Gerontology, 64A, 312-317.

Voukelatos, A., Cumming, R,G., Lord, S,R., \& Rissel, C. (2007) A randomized, controlled trial of tai chi for the prevention of falls: the Central Sydney tai chi trial. Journal of the American Geriatrics Society, 55(8), 1185-1191.

Ware, L. J., Epps, C. D., Herr, K., \& Packard, A. (2006) Evaluation of the Revised Faces Pain Scale, Verbal Descriptor Scale, Numeric Rating Scale, and lowa Pain Thermometer in Older Minority Adults. Pain Management Nursing, 7(3), 117-125.

Winter, D. A., Patla, A. E., \& Frank, J. S. (1990) Assessment of balance control in humans. Medical Progress through Technology, 16, 31-51.

Wolf, S. L., Barnhart, H. X., Kutner, N. G., McNeely, E., Coogler, C., \& Xu, T. (1996) Reducing frailty and falls in older persons: an investigation of Tai $\mathrm{Chi}$ and computerized balance training. Journal of the American Geriatrics Society, 44(5), 489-497.

World Health Organization (WHO). (2007) WHO Global Report on Falls Prevention in Older Age.

Zettergren, K. K., Lubeski, J. M., \& Viverito, J. M. (2011) Effects of a Yoga Program on Postural Control, Mobility, and Gait Speed in Community-Living Older Adults: A Pilot Study. Journal of Geriatric Physical Therapy, 34(2), 88-94. 
Figure 1: Participant flow chart

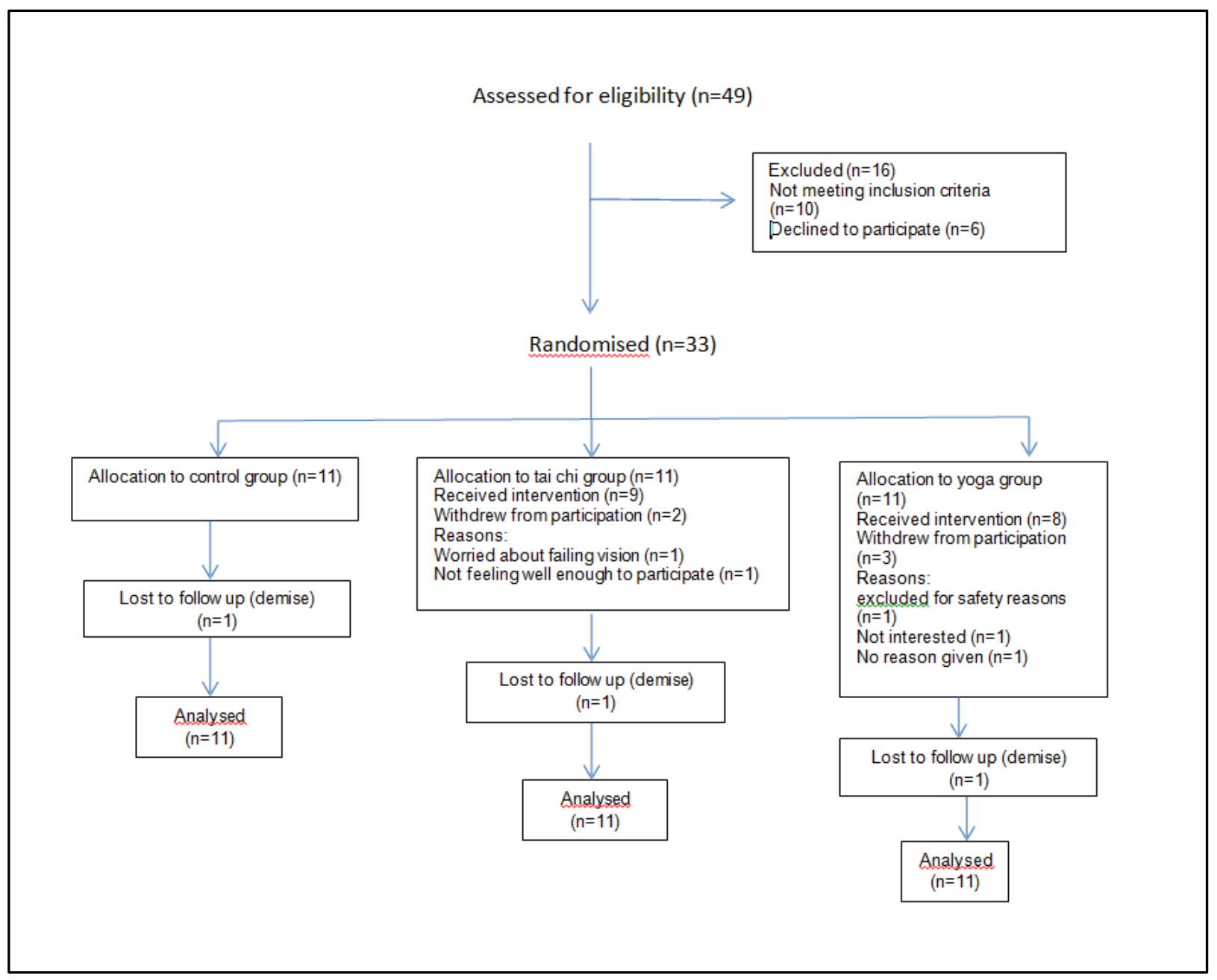


THE INFLUENCE OF TAI CHI AND YOGA ON BALANCE AND FALLS.

Table 1: Comparison of the control and intervention groups across a range of baseline characteristics.

\begin{tabular}{|c|c|c|c|c|c|c|}
\hline \multicolumn{2}{|c|}{ Baseline Characteristics } & \multirow{2}{*}{$\begin{array}{l}\text { Combined } \\
\text { (n=33) } \\
83.8(8.0)\end{array}$} & \multirow{2}{*}{$\begin{array}{c}\text { Tai Chi } \\
(n=11) \\
81.1(8.0)\end{array}$} & \multirow{2}{*}{$\begin{array}{c}\text { Yoga } \\
(n=11) \\
84.9(6.7)\end{array}$} & \multirow{2}{*}{$\begin{array}{l}\text { Control } \\
\text { (n=11) } \\
85.4(9.1)\end{array}$} & \multirow{2}{*}{$\begin{array}{c}\text { p-value } \\
0.3046\end{array}$} \\
\hline Age & mean (SD) & & & & & \\
\hline Gender & \%female & 72.7 & 72.7 & 90.9 & 54.5 & 0.1600 \\
\hline Mini Mental Score & mean(SD) & $25.9(2.6)$ & $25.7(2.5)$ & $27(2.4)$ & $25.2(2.7)$ & 0.2597 \\
\hline Number of co-morbidities & mean (SD) & $10.3(4.6)$ & $10.9(5.6)$ & $11.2(4.5)$ & $8.8(3.4)$ & 0.3712 \\
\hline Number of drugs consumed & mean (SD) & $13.1(5.1)$ & $14.6(5.8)$ & $12.7(5.3)$ & $12.1(4.2)$ & 0.5778 \\
\hline DEMQOL score at week 1 & mean (SD) & $92.3(13.7)$ & $89.7(19.1)$ & $93.9(7.1)$ & $93.5(12.7)$ & 0.8091 \\
\hline PAIN score at week 1 & mean (SD) & $1.8(1.9)$ & $1.2(1.4)$ & $2.5(1.9)$ & $1.7(2.3)$ & 0.2951 \\
\hline BBS score at week 1 & mean (SD) & $32.7(13.9)$ & $32.7(17.4)$ & $34(16.3)$ & $31.7(9.0)$ & 0.6792 \\
\hline Fall incidence ${ }^{*}$ & mean (SD) & $1.2(2.5)$ & $1.4(2.5)$ & $1.5(3.6)$ & $0.8(1.3)$ & 0.9932 \\
\hline
\end{tabular}

* During 6 months pre-intervention 
THE INFLUENCE OF TAI CHI AND YOGA ON BALANCE AND FALLS.

Table 2: Comparison of the control and intervention groups across a range of outcome measures.

\begin{tabular}{|c|c|c|c|c|c|c|c|c|c|}
\hline \multirow{2}{*}{\multicolumn{3}{|c|}{ Outcomes }} & \multicolumn{2}{|c|}{ Tai Chi group } & \multicolumn{2}{|c|}{ Yoga group } & \multicolumn{2}{|c|}{ Control group } & \multirow[t]{2}{*}{$p$ - value } \\
\hline & & & $\mathbf{n}$ & Mean (SD) & $\mathbf{n}$ & Mean (SD) & $\mathbf{n}$ & Mean (SD) & \\
\hline \multicolumn{3}{|c|}{ BBS score (week 1) } & 10 & $32.7(17.4)$ & 8 & $34.0(16.3)$ & 11 & $31.7(9.0)$ & \multirow{2}{*}{0.456} \\
\hline \multicolumn{3}{|c|}{ BBS score (week 14) } & 8 & $27.8(22.0)$ & 5 & $42.8(6.7)$ & 10 & $29.6(14.3)$ & \\
\hline \multicolumn{3}{|c|}{ Difference in BBS scores (week 14 - week 1) } & 8 & $-3.0(11.6)$ & 5 & $3.2(3.6)$ & 10 & $-3.4(11.2)$ & \\
\hline \multicolumn{3}{|c|}{ DEMQOL score (week 1) } & 11 & $89.7(19.1)$ & 10 & $93.9(7.0)$ & 11 & $93.5(12.7)$ & \multirow{3}{*}{0.945} \\
\hline \multicolumn{3}{|c|}{ DEMQOL score (week 7) } & 9 & $93.9(9.2)$ & 8 & $94.0(4.1)$ & 9 & $98.1(4.5)$ & \\
\hline \multicolumn{3}{|c|}{ DEMQOL score (week 14) } & 9 & $96.6(10.9)$ & 8 & $94.0(8.9)$ & 11 & $91.1(8.2)$ & \\
\hline \multirow{3}{*}{\multicolumn{2}{|c|}{$\begin{array}{l}\text { Difference in DEMQOL } \\
\text { scores }\end{array}$}} & weak 7 - week 1 & 9 & $4.2(15.0)$ & 8 & $0.0(4.6)$ & 9 & $2.8(10.7)$ & \\
\hline & & week 14 - week 1 & 9 & $6.9(18.0)$ & 8 & $0.0(6.5)$ & 11 & $-2.4(12.3)$ & \\
\hline & & week 14 - week 7 & 9 & $2.7(9.2)$ & 8 & $0.0(7.1)$ & 9 & $-6.8(7.4)$ & \\
\hline \multicolumn{3}{|c|}{ PAIN score (week 1) } & 11 & $1.2(1.4)$ & 10 & $2.5(1.9)$ & 11 & $1.7(2.3)$ & \multirow{3}{*}{0.436} \\
\hline \multicolumn{3}{|c|}{ PAIN score (week 7) } & 9 & $1.7(1.6)$ & 8 & $2.4(1.6)$ & 10 & $1.7(2.1)$ & \\
\hline \multicolumn{3}{|c|}{ PAIN score (week 14) } & 9 & $1.4(1.7)$ & 8 & $2.1(1.6)$ & 11 & $1.9(1.9)$ & \\
\hline \multirow{3}{*}{\multicolumn{2}{|c|}{ Difference in PAIN scores }} & week 7 - week 1 & 9 & $0.4(1.0)$ & 8 & $-0.4(2.8)$ & 10 & $-0.2(0.6)$ & \\
\hline & & week 14 - week 1 & 9 & $0.2(1.1)$ & 8 & $-0.6(3.1)$ & 11 & $0.2(2.2)$ & \\
\hline & & week 14 - week 7 & 9 & $-0.2(1.1)$ & 8 & $-0.3(2.3)$ & 10 & $0.2(2.2)$ & \\
\hline \multicolumn{3}{|c|}{$\begin{array}{l}\text { Fall incidence: } \\
\text { during } 6 \text { months pre-intervention }\end{array}$} & 11 & $1.4(2.5)$ & 11 & $1.5(3.6)$ & 11 & $0.8(1.3)$ & \multirow{3}{*}{0.648} \\
\hline \multicolumn{3}{|c|}{$\begin{array}{l}\text { Fall incidence: } \\
\text { during } 14 \text { week intervention }\end{array}$} & 9 & $0.0(0.0)$ & 9 & $0.7(0.9)$ & 11 & $0.4(0.5)$ & \\
\hline \multicolumn{3}{|c|}{$\begin{array}{l}\text { Fall incidence: } \\
\text { during 6months post intervention }\end{array}$} & 9 & $1.6(3.2)$ & 8 & $0.8(1.0)$ & 11 & $2.6(4.5)$ & \\
\hline \multirow{3}{*}{$\begin{array}{l}\text { Difference in fall } \\
\text { incidence }\end{array}$} & \multicolumn{2}{|c|}{ intervention - pre-intervention } & 9 & $-1.7(2.7)$ & 9 & $0.2(1.2)$ & 11 & $-0.5(1.5)$ & \\
\hline & \multicolumn{2}{|c|}{ post-intervention - pre-intervention } & 9 & $-0.1(2.1)$ & 8 & $0.3(0.9)$ & 11 & $1.8(5.0)$ & \\
\hline & \multicolumn{2}{|c|}{ post-intervention - intervention } & 9 & $1.6(3.2)$ & 8 & $0.1(1.6)$ & 11 & $2.3(4.3)$ & \\
\hline
\end{tabular}

* $p$-value obtained from generalized estimating equation (GEE) model DEMQOL: Dementia quality of life BBS: Berg Balance Scale 